BI-TP 2002/22

SWAT $/ 347$

\title{
The quark mass and $\mu$ dependence of the QCD chiral critical point *
}

\author{
Ch. Schmidt ${ }^{\mathrm{a}}$, C.R. Allton ${ }^{\mathrm{b}}$, S. Ejiri ${ }^{\mathrm{b}}$, S.J. Hands ${ }^{\mathrm{b}}$, O. Kaczmarek ${ }^{\mathrm{a}}$, F. Karsch ${ }^{\mathrm{a}}$ and E. Laermann ${ }^{\mathrm{a}}$ \\ ${ }^{\text {a} F a k u l t a ̈ t ~ f u ̈ r ~ P h y s i k, ~ U n i v e r s i t a ̈ t ~ B i e l e f e l d, ~ D-33615 ~ B i e l e f e l d, ~ G e r m a n y ~}$ \\ ${ }^{\mathrm{b}}$ Department of Physics, University of Wales Swansea, Singleton Park, Swansea, SA2 8PP, U.K.
}

In order to study the QCD chiral critical point we investigate Binder Cumulants of the chiral condensate. The results were obtained from simulations of 3 and $2+1$ flavors of standard staggered fermions and 3 flavors of p4 improved staggered fermions. The quark masses used are close to the physical quark mass. To extract the dependence on quark mass and chemical potential we apply a new reweighting technique based on a Taylor expansion of the action. The reweighting accuracy is $\mathcal{O}(m)$ for the standard and $\mathcal{O}\left(m^{2}\right), \mathcal{O}\left(\mu^{2}\right)$ for the p4 action.

\section{INTRODUCTION}

The phase transition in 3-flavor QCD is first order in the chiral limit $(m \equiv 0)$. This first order transition will persist for small but non-zero values of $m$ up to a critical value, $\bar{m}$, of the quark mass. QCD at this chiral critical point belongs to the universality class of the $3 \mathrm{~d}$ Ising model [1,2]. The generic phase diagram of QCD is shown in figure 1. In the space of two light quark masses

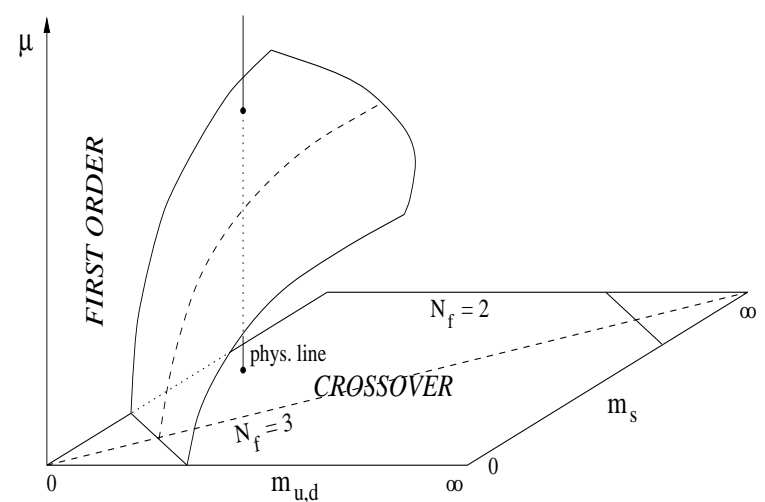

Figure 1. Generic phase diagram of QCD

\footnotetext{
*Presented by Ch. Schmidt. The work has been supported by the DFG under grant FOR 339/1-2 and by PPARC grant PPA/A/S/1999/00026.
}

$m_{u, d}$, one heavier quark mass $m_{s}$ and the quark chemical potential $\mu$, one expects a critical surface, which bends over the quark mass plane and separates the regime of first order phase transitions from the crossover regime.

To probe the order of the phase transition we compute the Binder Cumulant of the chiral condensate $B_{4}$ at $\mu=0$ for several quark masses at the corresponding critical coupling $\beta_{c}(m)$. Monte Carlo simulations for $\mu \neq 0$ are not possible due to the sign problem. $B_{4}$ is given by

$B_{4}=\frac{\left\langle(\delta \bar{\psi} \psi)^{4}\right\rangle_{\beta_{c}, m}}{\left\langle(\delta \bar{\psi} \psi)^{2}\right\rangle_{\beta_{c}, m}^{2}}, \quad \delta \bar{\psi} \psi=\bar{\psi} \psi-\langle\bar{\psi} \psi\rangle$.

This quantity is a renormalization group invariant quantity for $m=\bar{m}$ with a universal value, which depends on the universality class only. For the $3 \mathrm{~d}$ Ising model we have $B_{4}=1.604$ [3]. The critical surface is given by the surface of constant $B_{4}=$ 1.604. We define the critical coupling $\beta_{c}$ as the peak position of the chiral susceptibility.

\section{REWEIGHTING IN $m$ AND $\mu$}

In order to compute lines within the critical surface, we apply a reweighting technique based on a Taylor expansion [4,5]. Reweighting in couplings $w=m, \mu$ which couple to fermionic operators, is possible by introducing a reweighting 
factor $R$ in the expectation value

$$
\langle O\rangle_{w}=\left\langle O R\left(w, w_{0}\right)\right\rangle_{w_{0}} /\left\langle R\left(w, w_{0}\right)\right\rangle_{w_{0}} .
$$

Here the reweighting factor is given by

$$
\ln R=\ln \operatorname{det} Q(w)-\ln \operatorname{det} Q\left(w_{0}\right),
$$

with the fermion matrix $Q$. After expanding $\ln R$ one is left with

$$
\ln R=\sum_{n=1}^{\infty} \frac{\left(w-w_{0}\right)^{n}}{n !} R_{n}
$$

For fermionic observables like $\bar{\psi} \psi$ it is also necessary to expand the observable itself. For details see [4]. In addition we perform a reweighting in $\beta$ by the usual multi histogram reweighting, which is mandatory to follow the critical line and ensures the overlap of the configurations. Multiparameter reweighting was first applied to the problem of finite density QCD in [6].

\section{THE $m$ DEPENDENCE}

The 3-flavor critical point for standard staggered fermions was determined in 22]. To estimate the dependence of this critical point on non-degenerate quark masses, we performed $2+1$ flavor simulations for fixed $a m_{u, d}=0.03$ and $a m_{s}=0.045,0.06$. The volume is $12^{3} \times 4$. For each mass point we have three $\beta$-values. The total number of trajectories is 52000 for $a m_{s}=0.045$ and 7300 for $a m_{s}=0.06$. The results for the Binder Cumulants are shown in figure 2a. The straight line fit of $B_{4}$ together with the assumption of the 3d-Ising universality class, yields a critical strange quark mass. The critical values of the $2+1$, and 3 -flavor 2 calculations are listed in table 1 .

For mass reweighting the operator $R_{1}$ is the chiral condensate. We use the 3 -flavor data of mass

\begin{tabular}{|c|lcl|}
\hline flavor & \multicolumn{1}{|c}{$m_{u, d}$} & \multicolumn{1}{c|}{$m_{s}$} & \multicolumn{1}{c|}{$\beta_{\text {crit }}$} \\
\hline 3 & $0.0329(11)$ & $0.0329(11)$ & $5.1454(5)$ \\
$2+1$ & 0.0300 & $0.0391(50)$ & $5.1466(44)$ \\
\hline
\end{tabular}

Table 1

Critical points in the quark mass plane (standard action). The 3 -flavor result is from [2]
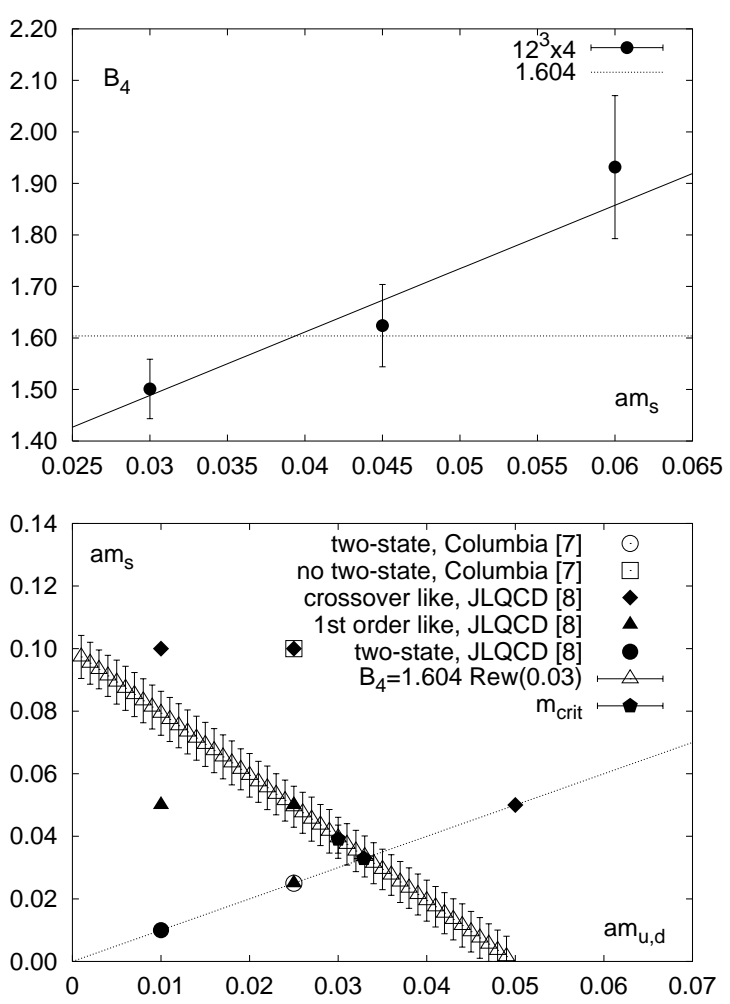

Figure 2. (a) Binder Cumulant of $(2+1)$-flavor QCD for the standard action and $a m_{u, d}=0.03$. The 3-flavor point is from [2]. (b) Line of second order phase transitions in the quark mass plane.

value $a m=0.03$ to compute the line of constant $B_{4}=1.604$ in the quark mass plane. The result is shown in figure $2 \mathrm{~b}$. The result is a straight line due to the reweighting order, for higher order reweighting one would expect deviations from a straight line. The validity range of the straight line is not known; nevertheless it is in agreement with the calculations of [7, 8 . The slope is -2 within errors, which is the expected value in the vicinity of the 3-flavor critical point.

\section{THE $\mu$ DEPENDENCE}

For 3-flavors of p4-improved fermions, a mass value of $a m=0.005$ and a volume of $12^{3} \times 4$, we 

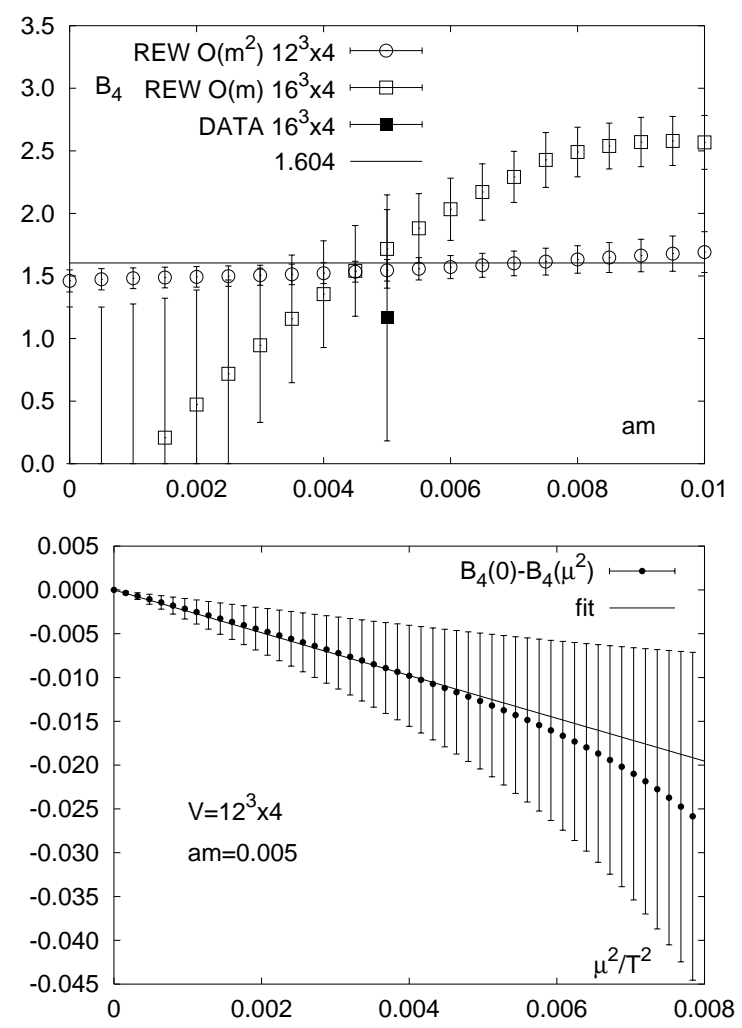

Figure 3. Binder Cumulant of 3-flavor QCD for the p4-improved action, (a) as a function of $m$, (b) as a function of $\mu^{2} / T^{2}$.

measured the reweighting operators $R_{1}, R_{2}$ for $m$ and $\mu$ reweighting. Here we have five $\beta$-values, with a total number of 6100 trajectories. For $a m=0.01$ and $V=16^{3} \times 4$ we use $R_{1}=\bar{\psi} \psi$ for mass reweighting. To determine the critical mass value $\bar{m}$, we compute $B_{4}$ as a function of $m$. The result is shown in figure $3 a$. The two different volumes have an intersection point near the value of $B_{4}=1.604$, i.e. 3d Ising universality class. Due to the large errors we give an upper bound for the critical mass only, which is $a \bar{m}<0.0075$, or in terms of the pion mass $\bar{m}_{P S}<190 \mathrm{MeV}$.

For increasing quark chemical potential $\mu_{u}=$ $\mu_{d}=\mu_{s}$ we find a decreasing Binder Cumulant, as shown in figure $3 \mathrm{~b}$ for the $12^{3} \times 4$ lat-

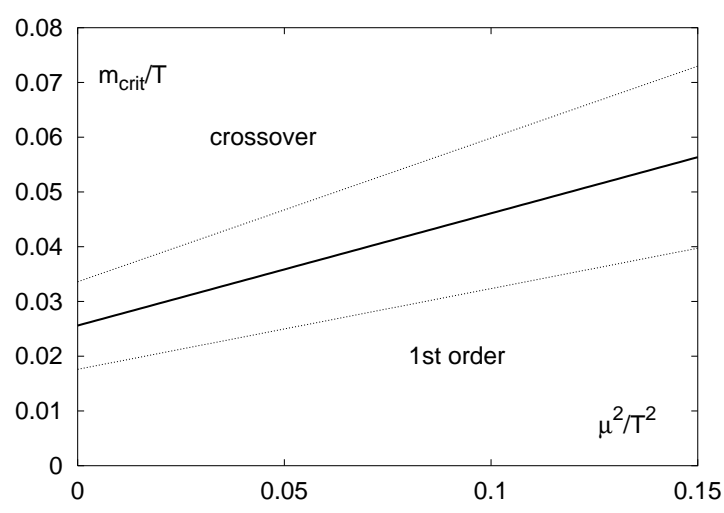

Figure 4. Sketch of the phase diagram. Dotted lines indicate the error band.

tice. From the two partial derivatives $\partial B_{4} / \partial(\mathrm{am})$ and $\partial B_{4} / \partial\left(a^{2} \mu^{2}\right)$, which we get from straight line fits of the reweighted data, and the assumption that $\partial B_{4} / \partial(\mathrm{am})$ is constant in $\mathrm{am}$, one can compute the quantity $\partial(a \bar{m}) / \partial\left(a^{2} \mu^{2}\right)$. The first derivatives $\partial B_{4} / \partial(a \mu)$ and $\partial(a \bar{m}) / \partial(a \mu)$ vanish because of symmetry reasons. A jackknife analysis yields $a^{-1} \partial \bar{m} / \partial\left(\mu^{2}\right)=0.82(23)$, or equivalent $T \partial \bar{m} / \partial\left(\mu^{2}\right)=0.21(6)$. The resulting phase diagram is sketched in figure 4 . The straight line corresponds to the curved dashed line in figure 1 .

\section{REFERENCES}

1. S. Gavin, A. Gocksch and R.D. Pisarski, Phys. Rev. D49 (1994) 3079.

2. F. Karsch, E. Laermann and Ch. Schmidt, Phys. Lett. B520 (2001) 41.

3. H.W. Blöte, E. Luijten and J.R. Heringa, J. Phys. A: Math. Gen. 28 (1995) 6289.

4. C.R. Allton et al., arXiv:hep-lat/0204010.

5. S. Ejiri et al., these proceedings.

6. Z. Fodor and S.D. Katz, Phys. Lett. B534 (2002) 87.

7. F.R. Brown et al., Phys. Rev. Lett. 65 (1990) 2491.

8. S. Aoki et al., Nucl. Phys. Proc. Suppl. 73 (1999) 459. 